KCL-PH-TH/2016-03

\title{
Linear stability of noncommutative spectral geometry
}

\author{
M. Sakellariadoua and A. Watcharangkoob \\ Department of Physics, King's College London, University of London, Strand WC2R 2LS, London, United Kingdom
}

We consider the spectral action within the context of a 4-dimensional manifold with torsion and show that, in the vacuum case, the equations of motion reduce to Einstein's equations, securing the linear stability of the theory. To subsequently investigate the nonvacuum case, we consider the spectral action of an almost commutative torsion geometry and show that the Hamiltonian is bounded from below, a result which guarantees the linear stability of the theory.

\footnotetext{
a email address: mairi.sakellariadou@kcl.ac.uk

b email address: apimook.watcharangkool@kcl.ac.uk
} 


\section{INTRODUCTION}

Consider the gravitational action

$$
S_{\mathrm{gr}}\left[g_{\mu \nu}\right]=\int \sqrt{|g|}\left(\bar{\Lambda}+\frac{1}{\kappa^{2}} R-\alpha_{0}\|C\|^{2}\right) d^{4} x,
$$

where $\bar{\Lambda}$ denotes the cosmological constant, $\kappa^{2}=16 \pi G, \alpha_{0}$ is a positive constant and $\|C\|^{2}:=C^{\mu \nu \rho \sigma} C_{\mu \nu \rho \sigma}$ is the Weyl invariant. This action, Eq. (1), belongs to a family of higher derivative theories, since it contains a fourth order derivative of the metric tensor, $g_{\mu \nu}$, namely $\left(\partial^{2} g\right)^{2}$. The presence of this higher derivative term may give rise to an unbounded (from below) Hamiltonian, implying the onset of a classical instability [1 3]. Gravitational theories with curvature invariants, as for instance shown in the action (1) above, belong to the class of nondegenerate higher derivative theories plagued by the Ostrogradski instability (linear instability). Such theories can appear naturally in the context of fundamental theories, as for instance within one-loop corrections of quantum theories on a curved background, or within the spectral action of almost commutative geometry. Despite the fact that theories with higher derivative terms may be pathological, they may instead improve the ultraviolet convergence of the graviton propagator within a linearized theory, rendering the theory power counting renormalizable [1].

The linear instability of a higher derivative nondegenerate theory may be removed, if one assumes the action as an effective one [4] and imposes appropriate constraints leading to a reduction of the trajectories of the degrees of freedom, hence rendering the effective Hamiltonian bounded from below. In this approach, a necessary but not sufficient condition in order to remove the instability, is that the imposed constraints must be such that they reduce the dimensionality of the original phase space [3]. A different approach has been suggested in Ref. [5], where one generalizes a higher derivative theory into an $\mathrm{SO}(4,2)$ gauge theory, and then derives conditions such that the equations of motion reduce, in some basis, to the vacuum Einstein's equations. As it has been shown [5], varying all of the connection fields, and not only the metric, Weyl gravity transforms from a fourth order theory into a theory of conformal equivalence classes of solutions to general relativity, under the requirement that torsion vanishes. In what follows, we show the linear stability of the spectral action for a 4-dimensional manifold with torsion and in the absence of any matter fields, adapting the approach proposed in Ref. [5]. We subsequently extend this approach in the nonvacuum case.

This paper is organized as follows. In Section 2, we briefly introduce [6 9 ] the concept of spectral geometry and spectral action. In Section 3, we review the approach discussed in Ref. [5], and apply it to the fourth order gravitational theory described by the action (11). We show that such theory does not suffer from linear instability. In Section 4, we consider the spectral action of an almost commutative torsion geometry and show that the obtained Hamiltonian is bounded from below, hence the theory does not suffer from linear instability even in the nonvacuum case. We round up our conclusions in Section 5.

\section{ELEMENTS OF THE SPECTRAL ACTION}

Consider a compact 4-dimensional Riemannian spin manifold $M$ and a spinor bundle $S \rightarrow M$. The set of smooth, infinitely differentiable, functions $C^{\infty}(M)$ forms an algebra $A$ under pointwise multiplication. This algebra acts on the Hilbert space of square-integrable spinors on $M, H=L^{2}(M, S)$, as multiplication operators. Then consider the Dirac operator $\mathcal{D}$, given in terms of the spin Levi-Civita connection $\nabla^{S}$ and the Dirac gamma matrices $\gamma^{\mu}$ as $-i \gamma^{\mu} \nabla_{\mu}^{S}$. The compact Riemannian spin manifold $M$ is fully described [10] by the canonical spectral triple $(A, H, \mathcal{D})$. Hence, spectral data can characterize the geometry of ordinary Riemannian manifolds, in the sense that the canonical spectral triple $(A, H, \mathcal{D})$ encodes the spacetime structure.

Let us extend the spectral triple approach for noncommutative manifolds. Conside the finite $C^{*}$-algebra

$$
A_{F}=\mathbb{C} \oplus \mathbb{H} \oplus M_{4}(\mathbb{C}),
$$

together with a finite dimensional Hilbert space $H_{F}$ and a self-adjoint operator $D_{F}$. The spectral triple $\left(A_{F}, H_{F}, D_{F}\right)$ can be identified with a finite space of points $F$. Although the finite spectral triple by itself gives an uninteresting structure, its product with the canonical spectral triple, namely

$$
\left(C^{\infty}(M) \otimes A_{F}, L^{2}(M, S) \otimes H_{F}, \not \nabla \otimes \operatorname{Id}_{F}+\gamma^{5} \otimes D_{F}\right),
$$

yields a nontrivial noncommutative structure [8]. The spectral triple (3) is called the almost commutative spectral triple. The canonical triple encodes the spacetime structure while the finite spectral triple encodes the internal degrees of freedom at each point of the 4-dimensional spacetime. The particle physics model one has in mind is encoded in 
the finite dimensional Hilbert space $H_{F}$. In the case of the Standard Model, the generalized Dirac operator acting on the Hilbert space $H=L^{2}(M, S) \otimes H_{F}$, contains the Higgs boson, Yukawa couplings, neutrino masses, as well as the Cabibbo-Kobayashi-Maskawa matrix.

The dynamics are given by a spectral action that sums up all frequencies of vibration of space. The spectral action is defined as the heat kernel trace of the operator $\mathcal{D}^{2}$ :

$$
S=\operatorname{Tr}_{L^{2}} f\left(\mathcal{D}^{2} / \Lambda^{2}\right)
$$

where $f$ is a positive cut-off function and $\Lambda$ a cut-off scale. For the canonical spectral triple the spectral action reads

$$
S \sim \int \sqrt{|g|}\left(\frac{f_{4}}{2 \pi^{2}} \Lambda^{4}+\frac{f_{2}}{24 \pi^{2}} \Lambda^{2} R-\frac{f(0)}{16 \pi^{2}}\|C\|^{2}\right) d^{4} x+\mathcal{O}\left(\Lambda^{-2}\right),
$$

where

$$
f_{4-k}=\int_{0}^{\infty} x^{4-k-1} f(x) d x \quad, \quad 0 \leq k<4 .
$$

Note that the action (5) is of the same form as the action (1), which is a higher derivative gravitational theory. In the case of the almost commutative spectral triple, the spectral action reads [8]

$$
\begin{aligned}
S \sim \int \sqrt{|g|}[ & \frac{48 f_{4}}{\pi^{2}} \Lambda^{4}-\frac{c f_{2}}{\pi^{2}} \Lambda^{2}+\frac{d f(0)}{4 \pi^{2}} \frac{4 f_{2}}{\pi^{2}} \Lambda^{2}-\frac{c f(0)}{24 \pi^{2}} R-\frac{3 f(0)}{10 \pi^{2}}\|C\|^{2} \\
& +\frac{1}{4} F_{\mu \nu} F^{\mu \nu}+\frac{1}{4} W_{\mu \nu}^{a} W^{\mu \nu, a}+G_{\mu \nu}^{i} G^{\mu \nu, i} \frac{1}{2}\left|\nabla_{\mu}^{\prime} H\right|^{2}-\frac{1}{12} R H^{2} \\
& \left.-\frac{2 a f_{2} \Lambda^{2}-e f(0)}{a f(0)} H^{2}+\frac{b \pi^{2}}{2 a^{2} f(0)} H^{4}\right] d^{4} x+\mathcal{O}\left(\Lambda^{-2}\right)
\end{aligned}
$$

where the action of $\nabla^{\prime}$ on the Higgs field is defined as

$$
\nabla_{\mu}^{\prime} H:=\partial_{\nu} H+\frac{1}{2} i g_{2} W_{\mu}^{a} \sigma^{a} H-\frac{1}{2} i g_{1} A_{\mu} H .
$$

and the constants $a, b, c, d$ and $e$ are derived from Yukawa mass matrices. The gauge fields $A_{\mu}, W_{\mu}$ and $G_{\mu}$ belong to the Lie algebra of the symmetry groups $\mathrm{U}_{Y}(1), \mathrm{SU}(2)$ and $\mathrm{SU}(3)$, respectively.

\section{FOURTH ORDER WEYL GRAVITY}

Consider the higher derivative theory

$$
S=\int \Omega_{B}^{A} \wedge^{*} \Omega_{A}^{B},
$$

where $\Omega_{B}^{A}$ stands for the $\mathrm{SO}(4,2)$ curvature 2-form. As it has been shown in Ref. [5], varying the above action with respect to the connection, the higher order equations of motion can be reduced, in the absence of torsion, to the vacuum second order Einstein's equations. The solutions are conformal equivalence metrics of Ricci-flat spacetimes. Following this approach for the generalized spectral action which is invariant under a smaller symmetry group, i.e. local Poincaré symmetry, we will show that in the absence of torsion the equations of motion combined with the Bianchi identity lead to an integrability condition that implies the reduction to the second order Einstein's equations.

To generalize the action Eq. (5) into a gauge theory with a Poincaré symmetry one needs to equip a manifold with a tetrad $e_{\mu}^{a}$,

$$
g_{\mu \nu}=\eta_{a b} e_{\mu}^{a} e_{\nu}^{b}
$$

and a spin connection $\omega_{\mu}^{a b} \in \mathfrak{s o}(1,3)$, satisfying

$$
D_{\mu} e_{\nu}^{a}:=\partial_{\mu} e_{\nu}^{a}-\Gamma_{\mu \nu}^{\alpha} e_{\alpha}^{a}+\omega_{\mu c}^{a} e_{\nu}^{c}=0,
$$

where latin characters denoting flat spacetime indices, $D_{\mu}$ is the covariant derivative and $\Gamma_{\mu \nu}^{\alpha}$ is an affine connection. The curvature two-form of the spin connection, defined by

$$
R_{\mu \nu}^{a b}:=\partial_{\mu} \omega_{\nu}^{a b}-\partial_{\nu} \omega_{\mu}^{a b}+\omega_{\mu c}^{a} \omega_{\nu}^{c b}-\omega_{\nu c}^{a} \omega_{\mu}^{c b}
$$


is independent of the tetrad basis. In general, the spin connection is not necessarily torsion-free. In fact, the curvature two-form (12) contains a torsion and its derivative. This can be shown by contracting Eq. (11) with $e^{\nu, b}$,

$$
\begin{aligned}
\omega_{\mu}^{a b} & =e_{\nu}^{a} e^{\sigma, b} \Gamma_{\mu \sigma}^{\nu}+e_{\nu}^{a} \partial_{\mu} e^{\nu, b} \\
& =e_{\nu}^{a} e^{\sigma, b}\left(\Gamma_{(\mu \sigma)}^{\nu}+\Gamma_{[\mu \sigma]}^{\nu}\right)+e_{\nu}^{a} \partial_{\mu} e^{\nu, b} \\
& =\left(e_{\nu}^{a} e^{\sigma, b} \Gamma_{(\mu \sigma)}^{\nu}+e_{\nu}^{a} \partial_{\mu} e^{\nu, b}\right)+e_{\nu}^{a} e^{\sigma, b} \Gamma_{[\mu \sigma]}^{\nu} \\
& =\left(e_{\nu}^{a} e^{\sigma, b} \Gamma_{(\mu \sigma)}^{\nu}+e_{\nu}^{a} \partial_{\mu} e^{\nu, b}\right)+\frac{1}{2} e_{\nu}^{a} e^{\sigma, b} T_{\mu}^{\nu},
\end{aligned}
$$

where $T_{\mu \sigma}^{\nu}:=2 \Gamma_{[\mu \sigma]}^{\nu}$ is the torsion tensor. The subscript notation "( )" denotes symmetrization $\Gamma_{(\mu \sigma)}^{\nu}:=\frac{1}{2}\left(\Gamma_{\mu \sigma}^{\nu}+\Gamma_{\sigma \mu}^{\nu}\right)$ and "[ ]" donotes anti-symmetrization $\Gamma_{[\mu \sigma]}^{\nu}:=\frac{1}{2}\left(\Gamma_{\mu \sigma}^{\nu}-\Gamma_{\sigma \mu}^{\nu}\right)$.

Defining

$$
\omega_{\mu}^{\prime a b}:=e_{\nu}^{a} e^{\sigma, b} \Gamma_{(\mu \sigma)}^{\nu}+e_{\nu}^{a} \partial_{\mu} e^{\nu, b},
$$

we note that $\omega^{\prime}$ is torsion-free and the curvature (12) can be rewritten as

$$
R_{\mu \nu}^{a b}=R_{\mu \nu}^{\prime a b}+\nabla_{\mu} T_{\nu}^{a b}-\nabla_{\nu} T_{\mu}^{a b}+T_{\mu c}^{a} T_{\nu}{ }^{c b}-T_{\nu c^{a}} T_{\mu}{ }^{c b},
$$

where $\nabla$ is a covariant derivative acting on a tensor $v_{\nu}{ }^{a}$ as

$$
\nabla_{\mu} v_{\nu}^{a}:=\partial_{\mu} v_{\nu}^{a}-\Gamma_{(\mu \nu)}^{\alpha} v_{\alpha}^{a}+\omega_{\mu c}^{a} v_{\nu}^{c}
$$

and $R_{\mu \nu}^{\prime a b}$ is the curvature two-form of the torsion-free spin connection $\omega_{\mu}^{\prime a b}$, defined by

$$
R_{\mu \nu}^{\prime a b}:=\partial_{\mu} \omega_{\nu}^{\prime a b}-\partial_{\nu} \omega_{\mu}^{\prime a b}+\omega_{\mu c}^{\prime a} \omega_{\nu}^{\prime c b}-\omega_{\nu c}^{\prime a} \omega_{\mu}^{\prime c b}
$$

Denoting by $\mathcal{T}$ the set of all torsion fields, we consider a particular subset $\mathcal{T}_{R} \subset \mathcal{T}$, so that the torsion fields $T_{\mu}{ }^{a b} \in \mathcal{T}_{R}$ satisfy the following properties:

- $T_{\mu}{ }^{a b}$ is antisymmetric in the $a, b$ indices, and hence Eq. (13) implies that $\omega_{\mu}^{\prime a b}$ is also antisymmetric in $a, b$, leading to metric compatibility, and $\omega_{\mu}^{\prime a b}$ is just the Levi-Civita connection. The reason for choosing totally antisymmetric torsion fields is the following: The general connection on the tangent bundle of a manifold is compatible with the Riemannian metric and has the same geodesics as the Levi-Cevita connection if and only if the connection is the sum of the Levi-Cevita connection and a totally antisymmetric tensor field [11], thus the torsion field is totally antisymmetric. - $T_{\mu}{ }^{a b}$ yields the curvature tensor with the same symmetric properties as the Riemmanian curvature tensor, i.e.

$$
\begin{gathered}
R_{\mu \nu \sigma \rho}=-R_{\nu \mu \sigma \rho}=R_{\nu \mu \rho \sigma} \\
R_{\mu \nu \sigma \rho}=R_{\sigma \rho \mu \nu}
\end{gathered}
$$

where $R_{\mu \nu \sigma \rho}=R_{\mu \nu}{ }^{a b} e_{\sigma, a} e_{\rho, b}$. Note that (18) holds for all torsion fields $T_{\mu}^{a b} \in \mathcal{T}$, while (19) is only valid for $T_{\mu}{ }^{a b} \in \mathcal{T}_{R}$. With the above properties of the torsion fields, the Gauss-Bonnet action takes the form we are familiar with in Riemannian geometry, namely

$$
\chi_{E}=\frac{1}{8 \pi^{2}} \int \sqrt{|g|}\left(R_{\mu \nu}^{a b} R_{a b}^{\mu \nu}-4 R_{\mu}^{a} R_{a}^{\mu}+R^{2}\right) d^{4} x
$$

We note that the above action (20) is not valid for the more general class of torsions studied in Ref. [12].

Let us also define a traceless tensor $C_{\mu \nu}^{a b}$, as

$$
C_{\mu \nu}^{a b}:=R_{\mu \nu}^{a b}-\left(e_{\mu}^{[a} R_{\nu}^{b]}-e_{\nu}^{[a} R_{\mu}^{b]}\right)+\frac{1}{3} R e_{\mu}^{[a} e_{\nu}^{b]}
$$

where $R_{\mu}^{a}:=R_{\mu \nu}^{a b} e_{b}^{\nu}$ and $R:=R_{\mu}^{a} e_{\mu}^{a}$. We can thus generalize the spectral action Eq. (5) as follows:

$$
S_{\mathrm{gr}}\left[e_{\mu}^{a}, \omega_{\nu}^{a b}\right]=\int e\left(\alpha_{2} \Lambda^{4}+\frac{1}{\kappa^{2}} R_{\mu \nu}^{a b} e_{a}^{\mu} e_{b}^{\nu}-\alpha_{0} C_{\mu \nu}^{a b} C_{a b}^{\mu \nu}\right) d^{4} x
$$


where $e$ is defined as $e:=\sqrt{\left|\operatorname{det}\left(e_{\mu}^{a} e_{a, \nu}\right)\right|}=\sqrt{|g|}$. For a torsion field $T_{\mu}^{a b} \in \mathcal{T}_{R}$, it can be shown that the linearized theory obtained from the action (22) is equivalent to the one derived by the spectral action with torsion [12] (see Appendix B). Hence, the action (22) is linearly stable if and only if the spectral action with torsion is linearly stable.

Let us now derive the equations of motion obtained from the generalized action (22). The variation of the spin connection and the tetrad give respectively,

$$
\begin{aligned}
D_{\mu} C_{a b}^{\mu \nu}-\frac{1}{2} T_{\mu \alpha}{ }^{\nu} C_{a b}^{\mu \alpha} & =-\frac{1}{4 \alpha_{0} \kappa^{2}} T_{\mu \alpha}^{\nu} e_{a}^{\mu} e_{b}^{\alpha}, \\
R_{\mu \nu}-\frac{1}{2} g_{\mu \nu} R & =2 \alpha_{0} \kappa^{2} \Theta_{\mu \nu}+\frac{\kappa^{2}}{2} \alpha_{2} \Lambda^{4} g_{\mu \nu},
\end{aligned}
$$

where $\Theta_{\mu \nu}:=C_{\mu \alpha}^{a b} C_{\nu}{ }^{\alpha}{ }_{a b}-\frac{1}{4} g_{\mu \nu} C_{\mu \nu}{ }^{a b} C^{\mu \nu}{ }_{a b}$. To recover Einstein's equations from Eq. (24) we need first to set the torsion equal to zero, so that the connection becomes the Levi-Civita one. Thus,

$$
\begin{aligned}
\nabla_{\mu} C_{a b}^{\prime \mu \nu} & =0 \\
R_{\mu \nu}^{\prime}-\frac{1}{2} g_{\mu \nu} R^{\prime} & =2 \alpha_{0} \kappa^{2} \Theta_{\mu \nu}^{\prime}+\frac{\kappa^{2}}{2} \alpha_{2} \Lambda^{4} g_{\mu \nu},
\end{aligned}
$$

where $\Theta_{\mu \nu}^{\prime}:=\left.\Theta_{\mu \nu}\right|_{T=0}$. Since $\Theta_{\mu \nu}^{\prime}$ becomes the energy momentum tensor of the Weyl curvature, and therefore vanishes identically in 4-dimensions [13], we recover Einstein's equations with a cosmological constant.

The vanishing divergence of the Weyl curvature, Eq. (25), leads to the integrability condition once combined with the trace of the Bianchi identity

$$
\nabla_{\mu} C_{\nu \rho \sigma}^{\prime \mu}+\left(\nabla_{\sigma} S_{\nu \rho}^{\prime}-\nabla_{\rho} S_{\nu \sigma}^{\prime}\right)=0
$$

where $S_{\mu \nu}^{\prime}:=\frac{1}{2}\left(R_{\mu \nu}^{\prime}-\frac{1}{6} g_{\mu \nu} R^{\prime}\right)$ denotes the Schouten tensor. In the basis $e_{\mu}^{a}$, we get

$$
\nabla_{\sigma} S_{\nu \rho}^{\prime}-\nabla_{\rho} S_{\nu \sigma}^{\prime}=0
$$

which however is not the well-known integrability condition. To get the familiar expression [16] we introduce a new basis $e_{\mu}^{a} \mapsto \tilde{e}_{\mu}^{a}:=e^{\xi} e_{\mu}^{a}$, where $\xi(x)$ is a real-value function. Note that the Bianchi identity holds in this new basis, but the covariant derivative of the Weyl tensor transforms as

$$
\tilde{\nabla}_{\mu} \tilde{C}^{\prime \mu}{ }_{\nu \rho \sigma}=e^{-2 \xi}\left(\nabla_{\mu} C_{\nu \rho \sigma}^{\prime \mu}-\partial_{\mu} \xi C^{\prime \mu}{ }_{\nu \sigma}\right)
$$

To get the integrability condition, we consider Eq. (27) in the basis $\tilde{e}_{\mu}^{a}$ and use Eq. (29) and the field equation (25), to obtain

$$
\begin{aligned}
0 & =\tilde{\nabla}_{\mu} \tilde{C}^{\prime \mu}{ }_{\nu \rho \sigma}+\left(\tilde{\nabla}_{\sigma} \tilde{S}_{\nu \rho}^{\prime}-\tilde{\nabla}_{\rho} \tilde{S}_{\nu \sigma}^{\prime}\right) \\
& =e^{-2 \xi}\left(\nabla_{\mu} C^{\prime \mu}{ }_{\nu \rho \sigma}-\partial_{\mu} \xi C^{\prime \mu}{ }_{\nu \rho \sigma}\right)+\left(\tilde{\nabla}_{\sigma} \tilde{S}_{\nu \rho}^{\prime}-\tilde{\nabla}_{\rho} \tilde{S}_{\nu \sigma}^{\prime}\right) \\
& =-\left(\partial_{\mu} \xi\right) e^{-2 \xi} C^{\prime \mu}{ }_{\nu \rho \sigma}+\left(\tilde{\nabla}_{\sigma} \tilde{S}_{\nu \rho}^{\prime}-\tilde{\nabla}_{\rho} \tilde{S}_{\nu \sigma}^{\prime}\right) \\
& =-\left(\partial_{\mu} \xi\right) \tilde{C}^{\prime \prime}{ }_{\nu \rho \sigma}+\tilde{\nabla}_{\sigma} \tilde{S}_{\rho \nu}^{\prime}-\tilde{\nabla}_{\rho} \tilde{S}_{\sigma \nu}^{\prime},
\end{aligned}
$$

where we have used that $e^{-2 \xi} C^{\prime \mu}{ }_{\nu \rho \sigma}=\tilde{C}^{\prime \mu}{ }_{\nu \rho \sigma}$. Hence, the original manifold is conformally equivalent to a Ricci flat manifold. In other words, there exists a basis $\hat{e}_{\mu}^{a}:=e^{\zeta} \tilde{e}_{\mu}^{a}$, equal to $\hat{e}_{\mu}^{a}=e^{\xi+\zeta} e_{\mu}^{a}$ such that

$$
\hat{S}_{\mu \nu}^{\prime}=0
$$

leading to vanishing Ricci tensor, $\hat{R}_{\mu \nu}^{\prime}=0$. Therefore, the equation of motion (25) is conformally equivalent to the vacuum Einstein's equations and the theory is not plagued by a linear instability. Defining $\bar{\chi}:=\xi+\zeta$, the Schouten tensor reads

$$
\hat{S}_{\mu \nu}^{\prime}=S_{\mu \nu}^{\prime}-\nabla_{\mu} \partial_{\nu} \bar{\chi}+\partial_{\mu} \bar{\chi} \partial_{\mu} \bar{\chi}-\frac{1}{2} g_{\mu \nu} \partial^{\alpha} \bar{\chi} \partial_{\alpha} \bar{\chi}
$$

and Eq. (31) is compatible with Eq. (26) providing the scalar field $\bar{\chi}$ satisfies

$$
\nabla_{\mu} \partial_{\nu} \bar{\chi}-\partial_{\mu} \bar{\chi} \partial_{\nu} \bar{\chi}-g_{\mu \nu}\left(\nabla_{\alpha} \partial^{\alpha} \bar{\chi}+\frac{1}{2} \partial^{\alpha} \bar{\chi} \partial_{\alpha} \bar{\chi}\right)=\frac{1}{4} \kappa^{2} \alpha_{2} \Lambda^{4} g_{\mu \nu}
$$

In conclusion, considering the variation of the full connection, the higher order differential equations reduce to Einstein's equations obtained from either Eq. (25) or from Eq. (26). 


\section{HAMILTONIAN ANALYSIS OF THE THEORY INTERACTING WITH MATTER FIELDS}

Let us now assume that the gravitational action is defined in a 4-dimensional globally hyperbolic manifold, of the structure $\mathbb{R} \times \Sigma$, where $\Sigma$ is a Cauchy surface, i.e. any curve parametrized by $t \in \mathbb{R}$ intersects $\Sigma$ only once [18]. Consequently, if one picks the time direction along a normal vector on a Cauchy surface, there is no closed time-like curve in the manifold. More importantly, the existence of a Cauchy surface at any instant of time allows us to define the Poisson bracket, which is important for setting the Hamiltonian formalism.

Global hyperbolicity also allows us to choose a coordinate system $\left\{t, x^{i}\right\}$ such that the spatial coordinates are orthogonal to the time coordinate, i.e. $g_{t i}=0$. Let us choose flat spacetime basis $\left\{\mathbf{e}^{0}, \mathbf{e}^{I}\right\}$ with $I \in\{1,2,3\}$, such that the time direction is preserved

$$
\mathbf{e}^{0}=e_{t}^{0} d t \quad \text { and } \quad \mathbf{e}^{I}=e_{i}^{I} d x^{i}
$$

In the previous section we have avoided the linear instability by conformally reducing the equations of motion (25) to the vacuum Einstein's equations. The same method can be extended to the nonvacuum case as long as $\mathcal{L}_{\text {matter }}$ is not a function of the spin connection, as for instance for the Lagrangian of a gauge field. Note however that there are matter fields whose Lagrangian depends on the spin connection, as for example

$$
\begin{aligned}
\mathcal{L}_{H} & =\frac{1}{2}\left|\nabla_{\mu}^{\prime} H\right|^{2}-\frac{1}{12} R H^{2}-\mu^{2} H^{2}+\lambda H^{4}, \\
\mathcal{L}_{\psi} & =i \bar{\psi}\left(e_{a}^{\mu} \gamma^{a} D_{\mu}-m\right) \psi,
\end{aligned}
$$

where $\nabla_{\mu}^{\prime} H=\partial_{\mu} H+\left[B_{\mu}, H\right]$ and $D_{\mu} \psi:=\left(\partial_{\mu}+\frac{1}{4} \omega_{\mu}^{a b} \Sigma_{a b}\right) \psi$, for $\Sigma_{a b}:=\frac{1}{2}\left(\gamma_{a} \gamma_{b}-\gamma_{b} \gamma_{a}\right)$. Such Lagrangians lead to the equations of motion

$$
\nabla_{\mu} C_{a b}^{\prime \mu \nu}=\left.\frac{\delta \mathcal{L}_{\text {matter }}}{\delta \omega_{\nu}^{a b}}\right|_{T=0} \neq 0
$$

In such a case one cannot get the integrability condition using the same trick as previously, and hence one cannot argue the cure of the linear instability following the approach of Section 2. To show that there is no instability we will check directly that the Hamiltonian is bounded from below.

Without loss of generality let us turn off the gauge fields and the cosmological constant since they do not depend on the spin connection. By adding the Higgs field and a massive fermionic field into the action (22) we get

$$
S_{\mathrm{gr}}\left[e_{\mu}^{a}, \omega_{\nu}^{a b}\right]+S_{H, \psi}=\int d^{4} x e\left(\frac{1}{\kappa^{2}} R_{\mu \nu}^{a b} e_{a}^{\mu} e_{b}^{\nu}-\alpha_{0} C_{\mu \nu}^{a b} C_{a b}^{\mu \nu}+\mathcal{L}_{H, \psi}\right) .
$$

The canonical momenta are

$$
\begin{aligned}
\pi_{c d}^{\beta} & =-4 \alpha_{0} C_{c d}^{t \beta}+\left(\frac{2}{\kappa^{2}}-\frac{H^{2}}{6}\right) e_{[c}^{t} e_{d]}^{\beta}, \\
p_{0}^{t} & =0, p_{I}^{i}=0,
\end{aligned}
$$

where $\pi_{c d}^{\beta}, p_{0}^{t}$ and $p_{I}^{i}$ stand for the canonical momenta of $\omega_{\beta}^{c d}, e_{t}^{0}$ and $e_{i}^{I}$, respectively. Notice that the map $\pi_{\beta}^{a b} \mapsto \partial_{t} \omega_{\beta}^{a b}$ is not invertible for an arbitrary choice of the spin connection, therefore the Hamiltonian is not well-defined. To construct a well-defined Hamiltonian, let us consider a subset of spin connections such that each element can be decomposed into $\omega_{\mu}^{a b}=\Omega_{\mu}^{a b}+\tilde{\omega}_{\mu}^{a b}$ and the following two conditions are satisfied:

i) $C_{\mu \nu}^{a b}=\partial_{\mu} \Omega_{\nu}^{a b}-\partial_{\nu} \Omega_{\mu}^{a b}+\Omega_{\mu c}^{a} \Omega_{\nu}^{c b}-\Omega_{\nu c}^{a} \Omega_{\mu}^{c b}$,

ii) $\left(\Omega_{[\mu}^{a c} \tilde{\omega}_{\nu] c}^{b}+\tilde{\omega}_{[\mu}^{a c} \Omega_{\nu] c}^{b}\right) e_{b}^{\mu} e_{a}^{\nu}=0$.

We will call $i$ ) and $i$ ) the "splitting conditions" since they make the scalar curvature independent of $\Omega_{\mu}^{a b}$. To see this we rewrite the curvature $R=R_{\mu \nu}^{a b} e_{a}^{\mu} e_{b}^{\nu}$ in terms of $\Omega$ and $\tilde{\omega}$. Thus,

$$
\begin{aligned}
R_{\mu \nu}^{a b}= & \partial_{\mu} \Omega_{\nu}^{a b}-\partial_{\nu} \Omega_{\mu}^{a b}+\Omega_{\mu c}^{a} \Omega_{\nu}^{c b}-\Omega_{\nu c}^{a} \Omega_{\mu}^{c b} \\
& +\partial_{\mu} \tilde{\omega}_{\nu}^{a b}-\partial_{\nu} \tilde{\omega}_{\mu}^{a b}+\tilde{\omega}_{\mu c}^{a} \tilde{\omega}_{\nu}^{c b}-\tilde{\omega}_{\nu c}^{a} \tilde{\omega}_{\mu}^{c b} \\
& -2\left(\Omega_{[\mu}^{a c} \tilde{\omega}_{\nu] c}^{b}+\tilde{\omega}_{[\mu}^{a c} \Omega_{\nu] c}^{b}\right) .
\end{aligned}
$$


Assuming the validity of the conditions $i$ ) and $i i$ ) above, the scalar curvature reads

$$
\begin{aligned}
R= & R_{\mu \nu}^{a b} e_{a}^{\mu} e_{b}^{\nu} \\
= & C_{\mu \nu}^{a b} e_{a}^{\mu} e_{b}^{\nu}+\left(\partial_{\mu} \tilde{\omega}_{\nu}^{a b}-\partial_{\nu} \tilde{\omega}_{\mu}^{a b}+\tilde{\omega}_{\mu c}^{a} \tilde{\omega}_{\nu}^{c b}-\tilde{\omega}_{\nu c}^{a} \tilde{\omega}_{\mu}^{c b}\right) e_{a}^{\mu} e_{b}^{\nu} \\
& -2\left(\Omega_{[\mu}^{a c} \tilde{\omega}_{\nu] c}^{b}+\tilde{\omega}_{[\mu}^{a c} \Omega_{\nu] c}^{b}\right) e_{a}^{\mu} e_{b}^{\nu} \\
= & \left(\partial_{\mu} \tilde{\omega}_{\nu}^{a b}-\partial_{\nu} \tilde{\omega}_{\mu}^{a b}+\tilde{\omega}_{\mu c}^{a} \tilde{\omega}_{\nu}^{c b}-\tilde{\omega}_{\nu c}^{a} \tilde{\omega}_{\mu}^{c b}\right) e_{a}^{\mu} e_{b}^{\nu} .
\end{aligned}
$$

Note that the considered subset of spin connections is not empty, since it contains connections of all conformal Ricci flat geometry. Moreover, the splitting conditions hold automatically in the linearized theory. To prove this statement, let $h_{\mu \nu}$ denote the metric perturbation. The condition $(i i)$ is clearly satisfied since $\Omega_{\mu}^{a}{ }_{c} \tilde{\omega}_{\nu}{ }^{c b} e_{a}^{\mu} e_{b}^{\nu}$ is of order higher than $O\left(h^{2}\right)$. For condition $(i)$ one chooses the transverse traceless metric perturbation $\bar{h}_{\mu \nu}$ which satisfies the Laplace equation

$$
\square \bar{h}_{\mu \nu}=0
$$

where $\square$ denotes the flat space d'Alembertian. The Weyl tensor is

$$
\begin{aligned}
C_{\mu \nu \sigma \rho} & =\frac{1}{2}\left(\partial_{\sigma} \partial_{\nu} \bar{h}_{\mu \rho}+\partial_{\rho} \partial_{\mu} \bar{h}_{\nu \sigma}-\partial_{\rho} \partial_{\nu} \bar{h}_{\mu \sigma}-\partial_{\sigma} \partial_{\mu} \bar{h}_{\nu \rho}\right) \\
& =\eta_{\mu \lambda} \partial_{\sigma} \bar{\Gamma}_{\nu \rho}^{\lambda}-\eta_{\mu \lambda} \partial_{\rho} \bar{\Gamma}_{\nu \sigma}^{\lambda}
\end{aligned}
$$

where $\bar{\Gamma}_{\nu \rho}^{\lambda}:=\frac{1}{2} \eta^{\lambda \mu}\left(\partial_{\nu} \bar{h}_{\rho \mu}+\partial_{\rho} \bar{h}_{\nu \mu}-\partial_{\mu} \bar{h}_{\nu \rho}\right)$. Then using the definition of the spin connection, one can rewrite the Weyl tensor in terms of derivative of $\Omega_{\mu}^{a b}$, and therefore the condition $(i)$ is satisfied.

Defining

$$
\Pi_{c d}^{\beta}:=\frac{\partial \mathcal{L}}{\partial\left(\partial_{t} \Omega_{\beta}^{c d}\right)} \quad \text { and } \quad \tilde{\pi}_{c d}^{\beta}:=\frac{\partial \mathcal{L}}{\partial\left(\partial_{t} \tilde{\omega}_{\beta}^{c d}\right)}
$$

where $\mathcal{L}$ is the Lagrangian density of the action (38), and assuming the splitting conditions, one can then show that

$$
\begin{aligned}
& \Pi_{c d}^{\beta}=-4 \alpha_{0} C_{c d}^{t \beta}, \\
& \tilde{\pi}_{c d}^{\beta}=2\left(\frac{1}{\kappa^{2}}-\frac{H^{2}}{12}\right) e_{[c}^{t} e_{d]}^{\beta} .
\end{aligned}
$$

From the definition of the canonical momentum we get the constraints $\Pi_{c d}^{t}=0$ and $\tilde{\pi}_{c d}^{t}=0$, which are primary first class constraints and can be solved using the gauge fixing conditions $\Omega_{t}^{a b}=0, g^{i j} D_{i} \Omega_{j}^{a b}=0$ and $\tilde{\omega}_{t}^{a b}=0, g^{i j} D_{i} \tilde{\omega}_{j}^{a b}=$ 0 . The remaining constraints

$$
\begin{aligned}
\phi_{0}^{t} & :=p_{0}^{t}=0, \\
\phi_{I}^{i} & :=p_{I}^{i}=0, \\
\phi_{c} & :=\Pi_{c d}^{i} e_{i}^{d}=0, \\
\varphi_{c}^{j} & :=\Pi_{c d}^{j} e_{0}^{d}-4 \alpha_{0} C_{c d}^{i j} e_{i}^{d}=0, \\
\phi_{c d}^{j} & :=\tilde{\pi}_{c d}^{j}-2\left(\frac{1}{\kappa^{2}}-\frac{H^{2}}{12}\right) e_{[c}^{t} e_{d]}^{j}=0 .
\end{aligned}
$$

are primary second class constraints, and are also obtained from the definition of the canonical momentum. (We refer the reader to Ref. [19] for more details on a constrained Hamiltonian system.)

In what follows, let $P, Q$ to stand for the canonical variables and the symbol " $\approx$ " to denote the equality holding on the surface spanned by all constraints, called the "constraint surface", in short. Imposing all constraints, the 
Hamiltonian reads

$$
\begin{aligned}
\mathcal{H}= & P_{I} \partial_{t} Q^{I}-\mathcal{L} \\
= & \Pi_{c d}^{i} \partial_{t} \Omega_{i}^{c d}+\tilde{\pi}_{c d}^{i} \partial_{t} \tilde{\omega}_{i}^{c d}+p_{c}^{\beta} \partial_{t} e_{\beta}^{c}+p_{H} \dot{H}+p_{\psi} \dot{\psi}-\mathcal{L} \\
\approx & -\frac{1}{8 \alpha_{0}} \Pi_{c d}^{i} \Pi_{i}^{c d}+\alpha_{0} C^{i j}{ }_{c d} C_{i j}{ }^{c d}-\left(\frac{1}{\kappa^{2}}-\frac{H^{2}}{12}\right) R_{i j}{ }^{c d} e_{c}^{i} e_{d}^{j}+\mathcal{H}_{H, \psi} \\
\approx & -\frac{1}{4 \alpha_{0}} \Pi^{i}{ }_{0 I} \Pi_{i}{ }^{0 I}+\alpha_{0} C^{i j}{ }_{I J} C_{i j}{ }^{I J}-\left[\frac{1}{8 \alpha_{0}} \Pi_{I J}^{i} \Pi_{i}^{I J}-2 \alpha_{0} C^{i j}{ }_{0 I} C_{i j}{ }^{0 I}\right] \\
& -\left(\frac{1}{\kappa^{2}}-\frac{H^{2}}{12}\right) R_{i j}{ }^{c d} e_{c}^{i} e_{d}^{j}+\mathcal{H}_{H, \psi} \\
\approx & -\frac{1}{4 \alpha_{0}} \Pi^{i}{ }_{0 I} \Pi_{i}{ }^{0 I}+\alpha_{0} C^{i j}{ }_{I J} C_{i j}{ }^{I J}-\left(\frac{1}{\kappa^{2}}-\frac{H^{2}}{12}\right) R_{i j}{ }^{c d} e_{c}^{i} e_{d}^{j}+\mathcal{H}_{H, \psi},
\end{aligned}
$$

where $p_{H}$ and $p_{\psi}$ are the canonical momenta of the scalar field and the fermion field, respectively. Note that the term $\left[\frac{1}{8 \alpha_{0}} \Pi_{I J}^{i} \Pi_{i}^{I J}-2 \alpha_{0} C^{i j}{ }_{0 I} C_{i j}{ }^{0 I}\right]$ vanish due to the symmetry (19) of the curvature tensor.

Denote the set of second class primary constraints by $\Phi^{A}:=\left\{\phi_{0}^{t}, \phi_{I}^{i}, \phi_{c}, \varphi_{c}^{j}, \phi_{c d}^{j}\right\}$ and define a new Hamiltonian density as

$$
\mathcal{H}_{\text {tot }}:=\mathcal{H}+u_{A} \Phi^{A}
$$

where $u_{A}$ are Lagrange multipliers. All constraints need to satisfy the consistency condition

$$
0 \approx \dot{\Phi}^{A}=\left\{\Phi^{A}, \mathbf{H}_{t o t}\right\}
$$

where $\mathbf{H}_{\text {tot }}=\int \mathcal{H}_{t o t} e \mathrm{~d}^{3} x$ on some equal time surface $\Sigma_{t}$. By imposing the consistency condition on the constraints $\phi_{c}, \varphi_{c}^{j}, \phi_{0}^{t}$ and $\phi_{I}^{i}$ one obtains the secondary constraint (the full details can be found in Appendix $\mathrm{C}$ )

$$
\begin{aligned}
\chi & :=\frac{1}{2 \alpha_{0}} \Pi_{0 I}^{k} \Pi_{k}^{0 I}+2 \alpha_{0} C_{i j}{ }^{l k} C^{i j}{ }_{l k}+i \bar{\psi}\left(\gamma^{I} e_{I}^{i} D_{i} \psi-2 m \psi\right)-2 \mu^{2} H^{2}+2 \lambda H^{4} \\
& =0
\end{aligned}
$$

Using the constraint (58) the Hamiltonian reads

$$
\begin{aligned}
\mathcal{H} & \approx 2 \alpha_{0} C^{i j l k} C_{i j l k}-\frac{1}{2} i \bar{\psi} \gamma^{I} e_{I}^{i} D_{i} \psi-\left(\frac{1}{\kappa^{2}}-\frac{H^{2}}{12}\right) R_{i j}{ }^{I J} e_{I}^{i} e_{J}^{j}+\frac{1}{2} g_{t t} p_{H}^{2}-\frac{1}{2} g^{i j} \partial_{i} H^{\dagger} \partial_{j} H \\
& \approx \mathcal{H}_{C^{2}}+\mathcal{H}_{\mathrm{GR}}
\end{aligned}
$$

where $\mathcal{H}_{C^{2}}$ and $\mathcal{H}_{\mathrm{GR}}$ are defined respectively as

$$
\begin{aligned}
\mathcal{H}_{C^{2}} & :=2 \alpha_{0} C^{i j l k} C_{i j l k}-\frac{1}{2} i \bar{\psi} \gamma^{I} e_{I}^{i} D_{i} \psi, \\
\mathcal{H}_{\mathrm{GR}} & :=-\left(\frac{1}{\kappa^{2}}-\frac{H^{2}}{12}\right) R_{i j}{ }^{I J} e_{I}^{i} e_{J}^{j}+\frac{1}{2} g_{t t} p_{H}^{2}-\frac{1}{2} g^{i j} \partial_{i} H^{\dagger} \partial_{j} H .
\end{aligned}
$$

It is easy to show that $\mathcal{H}_{C^{2}}$ is bounded from below, since the first term is positive definite and the second one is proportional to the Hamiltonian of a massless fermion. To show that $\mathcal{H}_{\mathrm{GR}}$ is also bounded from below, let us recall the gauge fixing condition $\omega_{t}^{a b}=0$, which implies $T_{t}^{a b}=0$, since torsion is independent of the Levi-Civita spin connection. Using Eq. (15) we deduce that

$$
\begin{aligned}
R_{t i}{ }^{0 I} & =R_{t i}^{\prime 0 I}+\nabla_{t} T_{i}^{0 I}-\nabla_{i} T_{t}^{0 I}+T_{t}{ }_{J}^{0} T_{i}{ }^{J I}-T_{i}^{0}{ }_{J} T_{t}{ }^{J I} \\
& =R_{t i}^{\prime 0 I}
\end{aligned}
$$

while for $T_{\mu}^{a b} \in \mathcal{T}_{R}$, the scalar curvature obtained by contracting Eq. (15) reads

$$
R=R^{\prime}-\|T\|^{2}
$$


Hence,

$$
\begin{aligned}
R_{i j}{ }^{I J} e_{I}^{i} e_{J}^{j} & =R-2 R_{t i}{ }^{0 I} e_{0}^{t} e_{I}^{i} \\
& =R^{\prime}-\|T\|^{2}-2 R_{t i}^{\prime}{ }^{0 I} e_{0}^{t} e_{I}^{i} \\
& =\left(R^{\prime}-2 R_{t i}^{\prime}{ }^{0 I} e_{0}^{t} e_{I}^{i}\right)-\left(3 T_{t}{ }^{I J} T^{t}{ }_{I J}+T_{i}{ }_{K}{ } T_{j}{ }^{K J} e_{I}^{i} e_{J}^{j}\right) \\
& =R_{i j}^{\prime}{ }^{I J} e_{I}^{i} e_{J}^{j}-T_{i}{ }^{I}{ }{ }^{T}{ }_{j}{ }^{K J} e_{I}^{i} e_{J}^{j},
\end{aligned}
$$

which implies that $\mathcal{H}_{\mathrm{GR}}$ can be rewritten as

$$
\mathcal{H}_{\mathrm{GR}} \approx-\left(\frac{1}{\kappa^{2}}-\frac{H^{2}}{12}\right) R_{i j}^{\prime I J} e_{I}^{i} e_{J}^{j}+\frac{1}{2} g_{t t} p_{H}^{2}-\frac{1}{2} g^{i j} \partial_{i} H^{\dagger} \partial_{j} H+\left(\frac{1}{\kappa^{2}}-\frac{H^{2}}{12}\right) T_{i}{ }_{K}^{I} T_{j}^{K J} e_{I}^{i} e_{J}^{j},
$$

with a prime ' refering to torsion-free quantities. Assuming the Higgs field does not exceed the Planck mass, i.e. $H^{2}<12 / \kappa^{2}$, and noting that for the metric of signature $(+,-,-,-)$,

$$
\begin{aligned}
T_{i j k} T^{l j k} & =g^{i l} g^{j m} g^{k n} T_{i j k} T_{l m n} \\
& =\sum_{i, j, k=1}^{3} g^{i i} g^{j j} g^{k k} T_{i j k} T_{i j k} \leq 0,
\end{aligned}
$$

one concludes that the last term in the r.h.s. of Eq. (65), which can be written as $\left(\frac{1}{\kappa^{2}}-\frac{H^{2}}{12}\right) T_{i j k} T^{l j k}$, is negative definite, and therefore, unbounded from below. In contrast, the first two terms in the r.h.s. of Eq. (65) are just the canonical Hamiltonian of the Palatini action in the presence of a scalar field interaction term [15], leading to the classical dynamics of the Einstein-Hilbert action in the present of scalar field. We hence conclude that $\mathcal{H}_{R}$ is bounded from below if and only if torsion vanishes.

Finally, let us check whether the result agrees with Section 2. In the vacuum case the constraint (58) becomes

$$
\chi:=\frac{1}{2 \alpha_{0}} \Pi_{0 I}^{k} \Pi_{k}^{0 I}+2 \alpha_{0} C_{i j}{ }^{l k} C^{i j}{ }_{l k}=0 .
$$

Since $\Pi_{0 I}^{k} \Pi_{k}^{0 I}$ and $C_{i j}{ }^{l k} C^{i j}{ }_{l k}$ are positive definite, the constraint (67) implies that both terms have to vanish, and hence the Hamiltonian reads

$$
\mathcal{H} \approx \mathcal{H}_{\mathrm{GR}} \approx-\frac{1}{\kappa^{2}} R_{i j}{ }^{c d} e_{c}^{i} e_{d}^{j} .
$$

Hence, the Hamiltonian does not depend on the Weyl tensor, in agreement with the fact that the vacuum case reduces to Einstein gravity. Clearly then this Hamiltonian will give the same dynamics as Einstein's equations in vacuum.

The above analysis can be easily applied in the spectral action. In the simple vacuum case and considering a torsion field $T_{\mu}{ }^{a b} \in \mathcal{T}_{R}$, the third order differential equations can be reduced to the second order Einstein's equations. Therefore, in this case the theory does not suffer from a linear instability. In the case of an almost commutative torsion geometry and considering only matter fields whose Lagrangian do not depend on the spin connection, one can still guarantee the stability of the theory employing the method discussed in Section 2. Moreover, if fermions and conformal invariant scalar fields are present, the linear stability will still hold provided the splitting conditions (41) and (42) are satisfied.

\section{CONCLUSIONS}

Noncommutative spectral geometry is a theoretical framework that can offer a purely geometric explanation for the Standard Model of particle physics. The gravitational sector of the theory has terms beyond the Einstein-Hilbert action and in particular it contains higher derivative terms. Hence, one may wonder whether this gravitational theory may be plagued by linear instabilities, namely the appearance of negative energy modes. We have addressed this question here in two steps.

We have first considered the simple vacuum case and shown that introducing a particular type of torsion, one can apply the method presented in Ref. [5] and reduce the fourth order differential equations in those of second order derived from vacuum General Relativity, if and only if the torsion field vanishes. We have then cosnidered the spectral action of an almost commutative torsion geometry. For this latter case we have shown that one cannot obtain the integrability condition in the presence of either fermion fields or scalar fields. We have however argued that there exists a class of almost commutative torsion geometry that leads to a Hamiltonian which is bounded from below and hence argued that the theory does not suffer from a local instability. 


\section{Appendix A: Spin connection}

The covariant derivative of a spinor $\psi$ or a tensor $V_{\nu}^{a}$ can be expressed through the spin connection $\omega_{\mu}^{a b}$ as

$$
\begin{aligned}
D_{\mu} V_{\nu}^{a} & =\partial_{\mu} V_{\nu}^{a}-\Gamma_{\mu \nu}^{\sigma} V_{\sigma}^{a}+\omega_{\mu b}^{a} V_{\nu}^{b} \\
D_{\mu} \psi & =\partial_{\mu} \psi+\frac{1}{4} \omega_{\mu}^{a b} \Sigma_{a b} \psi
\end{aligned}
$$

respectively, where $\Sigma_{a b}=\frac{1}{2}\left(\gamma_{a} \gamma_{b}-\gamma_{b} \gamma_{a}\right)$ and $\Gamma_{\mu \nu}^{\sigma}$ stands for the affine connection defined as

$$
\Gamma_{\mu \nu}^{\sigma}=e_{\nu, b} e_{a}^{\sigma} \omega_{\mu}^{a b}-e_{\nu, b} \partial_{\mu} e^{\sigma, b} .
$$

The latter, Eq. (A3), can be rewritten as $D_{\mu} e_{\nu}^{a}=0$, dubbed as the tetrad postulate. Note that the validity of the tetrad postulate does not require to assume

- metric compatibility: $\omega_{\mu}^{a b}+\omega_{\mu}^{b a}=0$

- torsion-free: $\Gamma_{[\mu \nu]}^{\sigma}=0$.

If the spin connection is metric compatible, then one can decompose the curvature two-form into an irreducible representation of an orthogonal group as follows

$$
R_{\mu \nu}^{a b}=C_{\mu \nu}^{a b}+\left(e_{\mu}^{[a} R_{\nu}^{b]}-e_{\nu}^{[a} R_{\mu}^{b]}\right)-\frac{1}{3} R e_{\mu}^{[a} e_{\nu}^{b]},
$$

where $R_{\mu}^{a}=R_{\mu \alpha}^{a b} e_{b}^{\alpha}$ and $R=R_{\mu \nu}^{a b} e_{a}^{\mu} e_{b}^{\nu}$. Hence, in the coordinate basis, one has

$$
\begin{aligned}
R_{\mu \nu \rho \sigma} & =R_{\mu \nu}^{a b} e_{a, \rho} e_{b, \sigma} \\
& =C_{\mu \nu}^{a b} e_{a, \rho} e_{b, \sigma}+\left(e_{\mu}^{[a} R_{\nu}^{b]}-e_{\nu}^{[a} R_{\mu}^{b]}\right) e_{a, \rho} e_{b, \sigma}-\frac{1}{3} R e_{\mu}^{[a} e_{\nu}^{b]} e_{a, \rho} e_{b, \sigma} \\
& =C_{\mu \nu \rho \sigma}+\left(g_{\mu[\rho} R_{\sigma] \nu}-g_{\nu[\rho} R_{\sigma] \mu}\right)-\frac{1}{3} R g_{\mu[\rho} g_{\sigma] \nu} .
\end{aligned}
$$

Assuming also that the spin connection is torsion-free, one concludes that $C_{\mu \nu \rho \sigma}, R_{\mu \nu}$ and $R$ become the Weyl tensor, the Ricci tensor and the Ricci scalar, respectively.

\section{Appendix B: Equivalent linearized actions}

We will show that the linearized theories obtained from (i) the action (22) and (ii) the spectral action with torsion [12], are equivalent. First let us write down the spectral action with torsion, which we will denote by $S_{\text {TS }}$. For a torsion tensor $T_{\mu \nu \sigma} \in \mathcal{T}_{R}$, we have by definition that

$$
0=R_{\mu \nu \rho \sigma}-R_{\rho \sigma \mu \nu}=\frac{1}{2}(d T)_{\mu \nu \rho \sigma}-\nabla_{\rho} T_{\sigma \mu \nu}+\nabla_{\sigma} T_{\rho \mu \nu},
$$

and

$$
0=R_{\mu \rho}-R_{\rho \mu}=g^{\nu \sigma}\left(R_{\mu \nu \rho \sigma}-R_{\rho \sigma \mu \nu}\right)=\nabla_{\sigma} T_{\rho \mu}^{\sigma} .
$$

Hence, the spectral action (modulo the Euler characteristic number) is reduced to

$$
\begin{aligned}
S_{\mathrm{TS}} & \sim f_{4} \Lambda^{4} a_{0}\left(\mathcal{D}^{2}\right)+f_{2} \Lambda^{2} a_{2}\left(\mathcal{D}^{2}\right)+f(0) a_{4}\left(\mathcal{D}^{2}\right) \\
& \sim \int \sqrt{|g|} d^{4} x\left(\alpha_{2} \Lambda^{4}+\frac{1}{\kappa^{2}}\left(R^{\prime}-\|T\|^{2}\right)-\alpha_{0}\left\|C^{\prime}\right\|^{2}\right) .
\end{aligned}
$$

Note that the torsion tensor $T_{\mu \nu \sigma}:=3 \tilde{T}_{\mu \nu \sigma}$, where $\tilde{T}_{\mu \nu \sigma}$ denotes the torsion defined in Ref. 12]. To compare $S_{\mathrm{gr}}$ with $S_{\mathrm{TS}}$, we will write explicitly the torsion terms which are contained in $S_{\mathrm{gr}}$. Consider the square of the traceless tensor 
$C_{\mu \nu}^{a b}$ defined in Eq. (A44):

$$
\begin{aligned}
\|C\|^{2}= & \left\|R_{\mu \nu \rho \sigma}\right\|^{2}-2\left\|R_{\mu \nu}\right\|+\frac{1}{3} R^{2} \\
= & \left\|R_{\mu \nu \rho \sigma}^{\prime}\right\|^{2}+\frac{1}{4}\|d T\|^{2}-\frac{1}{3} R^{\prime}\|T\|^{2}+4 B(T)+\frac{1}{3}\|T\|^{4} \\
& -2\left(\left\|R_{\mu \nu}^{\prime}\right\|+\frac{1}{3}\|T\|^{4}-\frac{1}{2} R^{\prime}\|T\|^{2}+2 B(T)\right) \\
& +\frac{1}{3}\left(R^{\prime 2}-2 R^{\prime}\|T\|^{2}+\|T\|^{4}\right) \\
= & \left\|C^{\prime}\right\|^{2}+\frac{1}{4}\|d T\|^{2},
\end{aligned}
$$

where $B(T):=-R_{\mu \nu}^{\prime} T^{\mu \sigma \rho} T_{\sigma \rho}^{\nu}+\frac{1}{4} R^{\prime}\|T\|^{2}$ and the curvature scalar $R$ is $R=R^{\prime}-\|T\|^{2}$. Substituting $\|C\|^{2}$ and $R$ in the action (22) we get

$$
S_{\mathrm{gr}}=\int \sqrt{|g|}\left[\alpha_{2} \Lambda^{4}+\frac{1}{\kappa^{2}}\left(R^{\prime}-\|T\|^{2}\right)-\alpha_{0}\left(\left\|C^{\prime}\right\|^{2}+\frac{1}{4}\|d T\|^{2}\right)\right] d^{4} x .
$$

Using Eq. (B1) we rewrite $\|d T\|^{2}$ as

$$
\begin{aligned}
\|d T\|^{2} & =(d T)_{\mu \nu \rho \sigma}(d T)^{\rho \sigma \mu \nu} \\
& =\left(-\nabla_{\rho} T_{\sigma \mu \nu}+\nabla_{\sigma} T_{\rho \mu \nu}\right)\left(-\nabla^{\mu} T^{\nu \rho \sigma}+\nabla^{\nu} T^{\mu \rho \sigma}\right) \\
& =4 \nabla_{\rho} T_{\sigma \mu \nu} \nabla^{\mu} T^{\nu \rho \sigma} \\
& =4 \nabla^{\mu}\left(T^{\nu \rho \sigma} \nabla_{\rho} T_{\sigma \mu \nu}\right)-4 T^{\nu \rho \sigma} \nabla^{\mu} \nabla_{\rho} T_{\sigma \mu \nu} \\
& =4 \nabla^{\mu}\left(T^{\nu \rho \sigma} \nabla_{\rho} T_{\sigma \mu \nu}\right)+4 T^{\nu \rho \sigma} \nabla_{\rho} \nabla^{\mu} T_{\sigma \mu \nu}-4 T^{\nu \rho \sigma}\left[\nabla^{\mu}, \nabla_{\rho}\right] T_{\sigma \mu \nu} \\
& =4 \nabla^{\mu}\left(T^{\nu \rho \sigma} \nabla_{\rho} T_{\sigma \mu \nu}\right)-8 T^{\nu \rho \sigma}\left(R_{\rho \sigma \alpha}^{\prime \mu}-\frac{1}{2} \delta_{\sigma}^{\mu} R_{\rho \alpha}^{\prime}\right) T_{\mu \nu}^{\alpha} .
\end{aligned}
$$

Note that to obtain the last line we have used that the divergent of a torsion field vanishes (Eq. (B2)) and the identity

$$
\left[\nabla_{\mu}, \nabla_{\nu}\right] V_{\rho \sigma \alpha}=R_{\mu \nu \rho}^{\prime \beta} V_{\beta \sigma \alpha}+R_{\mu \nu \sigma}^{\prime \beta} V_{\rho \beta \alpha}+R_{\mu \nu \alpha}^{\prime \beta} V_{\rho \sigma \beta}
$$

Thus, the action $S_{\mathrm{gr}}$ reads

$$
\begin{aligned}
S_{\mathrm{gr}} & =\int \sqrt{|g|}\left[\alpha_{2} \Lambda^{4}+\frac{1}{\kappa^{2}}\left(R^{\prime}-\|T\|^{2}\right)-\alpha_{0}\left\|C^{\prime}\right\|^{2}\right] d^{4} x+2 \int \sqrt{|g|} T^{\nu \rho \sigma}\left(R_{\rho \sigma \alpha}^{\prime \mu}-\frac{1}{2} \delta_{\sigma}^{\mu} R_{\rho \alpha}^{\prime}\right) T_{\mu \nu}^{\alpha} d^{4} x \\
& =S_{\mathrm{ST}}+2 \int \sqrt{|g|} T^{\nu \rho \sigma}\left(R_{\rho \sigma \alpha}^{\prime \mu}-\frac{1}{2} \delta_{\sigma}^{\mu} R_{\rho \alpha}^{\prime}\right) T_{\mu \nu}^{\alpha} d^{4} x .
\end{aligned}
$$

Since the terms in the integrand appearing in the r.h.s. of Eq. (B8) are of order $\mathcal{O}\left(\omega^{3}\right)$, they can be discarded in the linearized theory. Thus, the actions $S_{\mathrm{gr}}$ and $S_{\mathrm{TS}}$ lead to theories which are equivalent in linear order.

\section{Appendix C: Solving the constraints}

For a constrained Hamiltonian system, the time evolution of any phase space function $f(P(x), Q(x))$ is defined by the Poisson bracket of $f$ with the Hamiltonian:

$$
\begin{aligned}
\left\{f(x), \mathbf{H}_{\mathrm{tot}}\right\}= & \int d^{3} y\left\{f(x), e \mathcal{H}_{\mathrm{tot}}(y)\right\}_{x_{0}=y_{0}} \\
& \int d^{3} y d^{3} z\left(\frac{\partial f(x)}{\partial Q(z)} \frac{\partial e \mathcal{H}_{\text {tot }}(y)}{\partial P(z)}-\frac{\partial f(x)}{\partial P(z)} \frac{\partial e \mathcal{H} \operatorname{tot}(y)}{\partial Q(z)}\right)_{x_{0}=y_{0}} .
\end{aligned}
$$

The consistency condition requires that the constraints do not have a time evolution on the constraint surface. 
At this point let us make a remark that will be useful later. Denoting by $\Phi^{A}$ the set of second class primary constraints, one has

$$
\begin{aligned}
0 & =\dot{\Phi}^{A}=\left\{\Phi^{A}, e \mathcal{H}_{\text {tot }}\right\}=\left\{\Phi^{A}, e \mathcal{H}+e u_{B} \Phi^{B}\right\} \\
& =\left\{\Phi^{A}, e \mathcal{H}\right\}+e u_{B}\left\{\Phi^{A}, \Phi^{B}\right\}+u_{B}\left\{\Phi^{A}, e\right\} \Phi^{B} \\
& \approx e\left(\frac{1}{e}\left\{\Phi^{A}, e \mathcal{H}\right\}+u_{B}\left\{\Phi^{A}, \Phi^{B}\right\}\right),
\end{aligned}
$$

where $u_{B}$ stand for Lagrange multipliers. Hence, if the quantity $\left(\frac{1}{e}\left\{\Phi^{A}, e \mathcal{H}\right\}+u_{B}\left\{\Phi^{A}, \Phi^{B}\right\}\right)$ is weakly equal to zero, then the consistency condition is satisfied. From Eq. (C2) one may either obtain the Lagrange multiplier $u_{B}$ or a new constraint, which is not a linear combination of the primary constraints. This new constraint is called the secondary constraint and we will define it by $\chi=0$.

In what follows we will derive the constraint (58). Note that we use the identities

$$
\begin{aligned}
\delta e_{a}^{\mu} & =-e_{b}^{\mu} e_{a}^{\nu} \delta e_{\nu}^{b}, \\
\delta e & =e e_{a}^{\mu} \delta e_{\mu}^{a}=-e e_{\mu}^{a} \delta e_{a}^{\mu} .
\end{aligned}
$$

Let us first reduce the number of unknown Lagrange multipliers by imposing the consistency condition on the constraints $\phi_{c}=0$ and $\varphi_{c}^{j}=0$.

- $0=\dot{\phi}_{c}=\left\{\phi_{c}, e \mathcal{H}_{\text {tot }}\right\}$ :

Using Eq. C2 the consistency condition implies

$$
0 \approx\left\{\phi_{c}, \mathcal{H}\right\}+u_{t}^{0}\left\{\phi_{c}, \phi_{0}^{t}\right\}+u_{i}^{I}\left\{\phi_{c}, \phi_{I}^{i}\right\}+w_{j}^{a}\left\{\phi_{c}, \varphi_{a}^{j}\right\} .
$$

Contraction with $e_{t}^{c}=\left(e_{t}^{0}, 0,0,0\right)$ then yields

$$
\begin{aligned}
0 & \approx\left\{e_{t}^{c} \phi_{c}, \mathcal{H}\right\}+u_{t}^{0} e_{t}^{c}\left\{\phi_{c}, \phi_{0}^{t}\right\}+u_{i}^{I} e_{t}^{c}\left\{\phi_{c}, \phi_{I}^{i}\right\}+w_{j}^{a} e_{t}^{c}\left\{\phi_{c}, \varphi_{a}^{j}\right\} \\
& \approx\left\{e_{t}^{0} \phi_{0}, \mathcal{H}\right\}+u_{t}^{0}\left\{e_{t}^{0} \phi_{0}, \phi_{0}^{t}\right\}-u_{t}^{0} \phi_{0}\left\{e_{t}^{0}, \phi_{0}^{t}\right\}+u_{i}^{I}\left\{e_{t}^{0} \phi_{0}, \phi_{I}^{i}\right\}+w_{a}^{j} e_{t}^{0}\left\{\phi_{0}, \varphi_{j}^{a}\right\} \\
& \approx\left\{e_{t}^{0} \phi_{0}, \mathcal{H}\right\}+u_{t}^{0}\left\{e_{t}^{0} \phi_{0}, \phi_{0}^{t}\right\}+u_{i}^{I}\left\{e_{t}^{0} \phi_{0}, \phi_{I}^{i}\right\}+w_{j}^{a} e_{t}^{0}\left\{\phi_{0}, \varphi_{a}^{j}\right\} .
\end{aligned}
$$

- $0=\dot{\varphi}_{J}^{j}=\left\{\varphi_{J}^{j}, e \mathcal{H}_{t o t}\right\}$ :

$$
0 \approx\left\{\varphi_{J}^{j}, \mathcal{H}\right\}+u_{t}^{0}\left\{\varphi_{J}^{j}, \phi_{0}^{t}\right\}+u_{i}^{I}\left\{\varphi_{J}^{j}, \phi_{I}^{i}\right\}-u_{c}\left\{\phi_{c}, \varphi_{J}^{j}\right\} .
$$

Contraction with $e_{j}^{J}$ then yields

$$
0 \approx\left\{e_{j}^{J} \varphi_{J}^{j}, \mathcal{H}\right\}+u_{t}^{0}\left\{e_{j}^{J} \varphi_{J}^{j}, \phi_{0}^{t}\right\}+u_{i}^{I}\left\{e_{j}^{J} \varphi_{J}^{j}, \phi_{I}^{i}\right\}-u_{c} e_{j}^{J}\left\{\phi_{c}, \varphi_{J}^{j}\right\}
$$

Combining Eqs. (C6), (C8) and using $e_{j}^{J} \varphi_{J}^{j}=e_{t}^{0} \phi_{0}$, one gets

$$
u^{c} e_{j}^{J}=-w_{j}^{J} e_{t}^{c}
$$

Defining the scalar $C:=\frac{1}{3} w_{j}^{J} e_{J}^{j}$ one then obtains

$$
u^{a}=-C e_{t}^{a}, \quad w_{j}^{J}=C e_{j}^{J}
$$

As a consequence of (C10) the total Hamiltonian is reduced to

$$
\begin{aligned}
\mathcal{H}_{t o t} & =\mathcal{H}-C e_{t}^{a} \phi_{a}+C e_{j}^{J} \varphi_{J}^{j}+u_{t}^{0} \phi_{0}^{t}+u_{i}^{I} \phi_{I}^{i}+u_{j}^{a b} \phi_{a b}^{j} \\
& =\mathcal{H}+u_{t}^{0} \phi_{0}^{t}+u_{i}^{I} \phi_{I}^{i}+u_{j}^{a b} \phi_{a b}^{j} .
\end{aligned}
$$

Next, to obtain the constraint Eq. (58), we analyze the consistency of the constraints $\phi_{0}^{t}=0$ and $\phi_{I}^{i}=0$. 
- $0=\dot{\phi}_{0}^{t}=\left\{\phi_{0}^{t}, e \mathcal{H}_{\text {tot }}\right\}$ :

We have

$$
\begin{aligned}
0= & \frac{1}{e}\left\{\phi_{0}^{t}, e \mathcal{H}\right\}+u^{a}\left\{p_{0}^{t}, \phi_{a}\right\}+w_{j}^{a}\left\{p_{0}^{t}, \varphi_{a}^{j}\right\}+u_{j}^{a b}\left\{p_{0}^{t}, \phi_{a b}^{j}\right\} \\
\approx & \left\{p_{0}^{t}, \mathcal{H}\right\}+\frac{1}{e} \mathcal{H}\left\{p_{0}^{t}, e\right\}-2 u_{j}^{0 J}\left(\frac{1}{\kappa^{2}}-\frac{H^{2}}{12}\right)\left(e_{0}^{t}\right)^{2} e_{J}^{j} \\
\approx & \frac{1}{2 \alpha_{0}} e_{0}^{t} \Pi_{0 K}^{i} \Pi_{i}^{0 K}+\left\{p_{0}^{t}, \mathcal{H}_{H, \psi}\right\}-e_{0}^{t} \mathcal{H}-2 u_{j}^{0 J}\left(\frac{1}{\kappa^{2}}-\frac{H^{2}}{12}\right)\left(e_{0}^{t}\right)^{2} e_{J}^{j} \\
\approx & \frac{3}{4 \alpha_{0}} e_{0}^{t} \Pi_{0 K}^{i} \Pi_{i}^{0 K}-\alpha_{0} e_{0}^{t} C_{i j}{ }^{I J} C^{i j}{ }_{I J}+\left(\frac{1}{\kappa^{2}}-\frac{H^{2}}{12}\right) e_{0}^{t} R_{i j}^{I J} e_{I}^{i} e_{J}^{j} \\
& +\left(\left\{p_{0}^{t}, \mathcal{H}_{H, \psi}\right\}-e_{0}^{t} \mathcal{H}_{H . \psi}\right)-2 u_{j}^{0 J}\left(\frac{1}{\kappa^{2}}-\frac{H^{2}}{12}\right)\left(e_{0}^{t}\right)^{2} e_{J}^{j} .
\end{aligned}
$$

Multiplying the above equation, Eq. (C12), with $e_{t}^{0}$ we obtain

$$
\begin{aligned}
\approx & \frac{3}{4 \alpha_{0}} \Pi_{0 K}^{i} \Pi_{i}^{0 K}-\alpha_{0} C_{i j}{ }^{I J} C^{i j}{ }_{I J}+\left(\frac{1}{\kappa^{2}}-\frac{H^{2}}{12}\right) R_{i j}^{I J} e_{I}^{i} e_{J}^{j} \\
& +\left(e_{t}^{0}\left\{p_{0}^{t}, \mathcal{H}_{H, \psi}\right\}-\mathcal{H}_{H . \psi}\right)-2 u_{j}^{0 J}\left(\frac{1}{\kappa^{2}}-\frac{H^{2}}{12}\right) e_{0}^{t} e_{J}^{j} .
\end{aligned}
$$

- $0=\dot{\phi}_{K}^{k}=\left\{\phi_{K}^{k}, e \mathcal{H}_{t o t}\right\}$ :

We have

$$
\begin{aligned}
0= & \frac{1}{e}\left\{p_{K}^{k}, e \mathcal{H}\right\}+u^{a}\left\{p_{K}^{k}, \phi_{a}\right\}+w_{j}^{a}\left\{p_{K}^{k}, \varphi_{a}^{j}\right\}+u_{j}^{a b}\left\{p_{K}^{k}, \phi_{a b}^{j}\right\} \\
\approx & \left\{p_{K}^{k}, \mathcal{H}\right\}+\frac{1}{e} \mathcal{H}\left\{p_{K}^{k}, e\right\}-2 u_{j}^{0 J}\left(\frac{1}{\kappa^{2}}-\frac{H^{2}}{12}\right) e_{0}^{t} e_{J}^{k} e_{K}^{j} \\
\approx & \frac{1}{2 \alpha_{0}} \Pi_{0 I}^{k} \Pi_{j}^{0 I} e_{K}^{j}+4 \alpha_{0} e_{K}^{m} C^{k l}{ }_{I J} C_{m l}^{I J}-2\left(\frac{1}{\kappa^{2}}-\frac{H^{2}}{12}\right) R_{i j}{ }^{I J} e_{I}^{i} e_{J}^{k} e_{K}^{j} \\
& +\left\{p_{K}^{k}, \mathcal{H}_{H, \psi}\right\}-e_{K}^{k} \mathcal{H}-2 u_{j}^{0 J}\left(\frac{1}{\kappa^{2}}-\frac{H^{2}}{12}\right) e_{0}^{t} e_{J}^{k} e_{K}^{j} \\
\approx & \frac{1}{2 \alpha_{0}} \Pi_{0 I}^{k} \Pi_{j}^{0 I} e_{K}^{j}+\frac{1}{4 \alpha_{0}} e_{K}^{k} \Pi_{0 I}^{i} \Pi_{i}^{0 I}+4 \alpha_{0}\left(e_{K}^{m} C^{k l}{ }_{I J} C_{m l}^{I J}-\frac{1}{4} e_{K}^{k} C^{i j}{ }_{I J} C_{i j}{ }^{I J}\right) \\
& -2\left(\frac{1}{\kappa^{2}}-\frac{H^{2}}{12}\right)\left(R_{i j}{ }^{I J} e_{I}^{i} e_{J}^{k} e_{K}^{j}-\frac{1}{2} e_{K}^{k} R_{i j}{ }^{I J} e_{I}^{i} e_{J}^{j}\right)+\left\{p_{K}^{k}, \mathcal{H}_{H, \psi}\right\}-e_{K}^{k} \mathcal{H}_{H, \psi} \\
& -2 u_{j}^{0 J}\left(\frac{1}{\kappa^{2}}-\frac{H^{2}}{12}\right) e_{0}^{t} e_{J}^{k} e_{K}^{j} .
\end{aligned}
$$

Contracting with $e_{k}^{K}$ we obtain

$$
\begin{aligned}
0 \approx & \frac{5}{4 \alpha_{0}} \Pi_{0 I}^{k} \Pi_{k}^{0 I}+\alpha_{0} C_{i j}{ }^{I J} C^{i j}{ }_{I J}+\left(\frac{1}{\kappa^{2}}-\frac{H^{2}}{12}\right) R_{i j}{ }^{I J} e_{I}^{i} e_{J}^{j} \\
& +e_{k}^{K}\left\{p_{K}^{k}, \mathcal{H}_{H, \psi}\right\}-3 \mathcal{H}_{H, \psi}-2 u_{j}^{0 J}\left(\frac{1}{\kappa^{2}}-\frac{H^{2}}{12}\right) e_{0}^{t} e_{J}^{j} .
\end{aligned}
$$

Combining Eqs. (C15) and Eq. C13 we have a constraint equation

$$
\begin{aligned}
0 \approx & \frac{1}{2 \alpha_{0}} \Pi_{0 I}^{k} \Pi_{k}^{0 I}+2 \alpha_{0} C_{i j}{ }^{l k} C^{i j}{ }_{l k}+\frac{1}{4 \alpha_{0}} \Pi_{I J}^{k} \Pi_{k}^{I J}+4 \alpha_{0} C_{i j}{ }^{0 I} C^{i j}{ }_{0 I} \\
& +e_{k}^{K}\left\{p_{K}^{k}, \mathcal{H}_{H, \psi}\right\}-e_{t}^{0}\left\{p_{0}^{t}, \mathcal{H}_{H, \psi}\right\}-2 \mathcal{H}_{H, \psi} \\
\approx & \frac{1}{2 \alpha_{0}} \Pi_{0 I}^{k} \Pi_{k}^{0 I}+2 \alpha_{0} C_{i j}{ }^{l k} C^{i j}{ }_{l k}+i \bar{\psi}\left(\gamma^{I} e_{I}^{i} D_{i} \psi-2 m \psi\right)-2 \mu^{2} H^{2}+2 \lambda H^{4} \\
= & : \chi,
\end{aligned}
$$


which is not a linear combination of the primary constraints. In conclusion, $\chi=0$ is a secondary constraint, which arises from the consistency condition.

[1] K. S. Stelle, Phys. Rev. D 16, 953 (1977). doi:10.1103/PhysRevD.16.953

[2] H. J. Schmidt, Phys. Rev. D 49, 6354 (1994).

[3] T. J. Chen, M. Fasiello, E. A. Lim and A. J. Tolley, JCAP 1302, 042 (2013) arXiv:1209.0583 [hep-th]].

[4] J. Z. Simon, Phys. Rev. D 41, 3720 (1990).

[5] J. T. Wheeler, Phys. Rev. D 90, no. 2, 025027 (2014) arXiv:1310.0526 [gr-qc]].

[6] A. Connes, Noncommutative Geometry, Academic Press, New York (1994).

[7] A. Connes and M. Marcolli, Noncommutative Geometry, Quantum Fields and Motives, Hindustan Book Agency, India (2008).

[8] W. D. van Suijlekom, "Noncommutative geometry and particle physics," Mathematical Physics Studies, Springer 2015, (published online)

[9] M. Sakellariadou, PoS CORFU 2011, 053 (2011) [arXiv:1204.5772 [hep-th]].

[10] A. Connes, J. Noncom. Geom. 7, 1, (2013).

[11] F. Hanisch, F. Pfaeffle, C. A. Stephan, Commun. Math. Phys. 300, 877 (2010).

[12] F. Pfaeffle, C. A. Stephan, J. Functional Analysis, 262, 1529 (2012).

[13] Trujillo, Juan Teancum, "Weyl Gravity as a Gauge Theory" (2013). http://digitalcommons.usu.edu/etd/1951

[14] J. F. Donoghue, Phys. Rev. D 40, 3874 (1994).

[15] M. Han, Y. Ma, Y. Ding, L. Qin, Mod. Phys. Lett. A 20, 725 (2005).

[16] P. Szekeres, Proc. R. Soc. A 274, 206 (1963).

[17] A. H. Chamseddine, A. Connes, M. Marcolli, Adv. Theor. Math. Phys. 11, 991 (2007).

[18] O. Müller, M. Sánchez, Jahresbericht der Deutschen Mathematiker-Vereinigung 1 (2013) arXiv:math/0604265v2 [math.DG], (2014).

[19] M. Henneaux, C. Teitelboim, "Quantization of Gauge Systems," Princeton University Press, 1992. 